[7] P. Henrici, "Upper bounds for the abscissa of stability of a stable polynomia]," SIAM J. Numer. A ral, vol. 7, pp. 538-544, 1970.

[8] S. Barnett and D. D. Siljak, "Routh's algorithm: A centennial survey," SIAM Rev., vol. 19, pp. $472-489,1977$.

[9] M. Mansour, E. I. Jury, and L. F. Chapparo, "Estimation of the margin of stability

[10] F. R. Gantmacher, The Theory of Matrices, vol. 2 . New York: Chelsea, 1960.

111] P. Henrici, Applied and Computational Anabosis, vol. 1. New York: Wiley, 1974.

[11] P. Henrici, Applied and Compusational Anthis, G. W. Stewart, "Error analysis of the algorithm for shifting the zeros
nomial by synthetic division," Math. Comp., vol. 25, pp. 135-139, 1971.

[13] C. E. Froberg, Introduction to Numerical Anabysis. Reading, MA: AddisonWesley, 1965.

[14] A. Van Der Sluis, "Upperbounds for roots of polynomials," Numer. Math, vol. 15, pp. 250-262, 1970.

[15] E. I. Jury, "Stability tests for one, two and multidimensional linear systems," Proc. Inst. Elec. Eng., vol. 124, pp. 1237-1240, Dec. 1977.

\section{Robustness with Observers}

\section{J. C. DOYLE, STUDENT MEMBER, IEEE, AND G. STEIN, MEMBER, IEEE}

Abstract-This paper describes an adjustment procedure for observerbased linear control systems which asymptotically achieves the same loop transfer functions (and hence the same relative stability, robustness, and disturbance rejection properties) as full-state feedback control implementations.

\section{INTRODUCTION}

The trouble with observers is that they tempt us, through the expediency of state reconstruction, to assign undue generality to control results proven only for the full-state feedback case. An example is the recent robustness result of Safonov and Athans [1]. This result shows that multivariable linear-quadratic optimal regulators have impressive robustness properties, including guaranteed classical gain margins of -6 $\mathrm{dB}$ to $+\infty \mathrm{dB}$ and phase margins of $+60^{\circ}$ in all channels. The result is only valid, however, for the full-state case. If observers or Kalman filters are used in the implementation, no guaranteed robustness properties hold. In fact, a simple example has shown that legitimate LQG controller-filter combinations exist with arbitrarily small gain margins in both the positive and negative $\mathrm{dB}$ direction [2].

In light of these observations, the robustness properties of control systems with filters or observers need to be separately evaluated for each design. Moreover, because such evaluations can come up with embarrassingly small margins, a "design adjustment procedure" to improve robustness would be very desirable. The present paper provides such a procedure. We show that while the commonly suggested approach of "speeding-up" observer dynamics will not work in general, alternate procedures which drive some observer poles toward stable plant zeros and the rest toward infinity do achieve the desired objective. In effect, full-state loop transfer properties can be recovered asymptotically if the plant is minimum phase. This occurs at the expense of noise performance.

The principal results of the paper are summarized in Section II, where we introduce and interpret certain transfer function properties of observer-based control systems, and in Section III, where we develop the "adjustment procedure." A simple example which illustrates these results is given in Section IV.

Manuscript received May 17, 1978; revised December 27, 1978. Paper recommended by M. K. Sain, Past Chairman of the Linear Systems Committee.

J. C. Doyle is with the Honeywell Systems and Research Center, Minneapolis, MN 55413 .

G. Stein is with the Honeywell Systems and Research Center, Minneapolis, MN 55413, and with the Department of Electrical Engineering and Computer Sciences, Massachusetts Institute of Technology, Cambridge, MA 02139.

\section{Transfer Function Properties of Observer-Based CONTROLLERS}

We consider the general multivariable control loop illustrated in Fig. 1. The plant is an $n$th order linear system, both observable and controllable, with $m$ inputs, $p=m$ outputs, and no transmission zeros [3] in the right half-plane. The control law consists of two transfer function matrices $H_{1}(s)$ and $H_{2}(s) . H_{2}$ is driven either with full-state feedback [Fig. 1(a)] or with an $n$th order observer [4] which reconstructs the state in the usual asymptotic sense [Fig. l(b)]. It is clear that this overall control loop includes linear-quadratic-Gaussian controllers as special cases. It also allows dynamic elements such as integrators and lag elements which may be required in more realistic control situations.

This configuration also applies to nonsquare plants for which the number of controls $m$ is not equal to the number of measurements $p$. For the case $m<p$, simply augment the original control vector with $(p-m)$ more components which are not driven by the controllers (i.e., $H_{1}^{T}=$ $\left[H_{11}^{T}: 0\right]$ ). Columns of the $B$ matrix for these added components must, of course, be selected to introduce no unstable transmission zeros. For the case $m>p$, select any $p$-dimensional subset of controls for which there are no right half-plane transmission zeros. Then the loop transfer properties which are established in this paper apply to this $p$-dimensional subset of control loops, with the remaining $(m-p)$ loops closed.

A dashed line is shown in both Fig. 1(a) and (b) in order to distinguish between elements of the loop which are part of the controller and those which are part of the plant. Since we design and implement the controller, there is relatively little uncertainty associated with it, whereas there may be significant differences between the actual plant and its model. The loop transfer functions which we examine for robustness, below, are then taken with respect to the loop breaking point $x$, at the control signal interface between these two sets of elements. Very misleading robustness results can be obtained for alternate loop breaking points, for example point $\times \times$. This is also shown below.

The following properties can be established for the above two controlloop implementations.

Property 1: The closed-loop transfer function matrices from command $r$ to state $x$ are identical in both implementations.

Property 2: The loop transfer function matrices from control signal $u^{\prime}$ to control signal $u$ (loops broken $\times \times$ ) are identical in both implementations.

Property 3: The loop transfer functions from control signal $u^{\prime \prime}$ to control signal $u^{\prime}$ (loops broken at point $X$ ) are generally different in the two implementations. They are identical if the observer dynamics satisfy

$$
K\left[I+C(s I-A)^{-1} K\right]^{-1}=B\left[C(s I-A)^{-1} B\right]^{-1}
$$

for all values of the complex variable $s$. The $A, B$, and $C$ 's above are plant matrices and $K$ is the observer gain.

The first two of these properties are very well known [5], [6]. They can be easily verified by noting that the transfer functions from $u^{\prime}$ to $x$ and from $u^{\prime}$ to $\hat{x}$ are identical because the nominal error dynamics of the observer are not controllable from $u^{\prime}$. Hence, the error dynamics are not excited by inputs $r$ to the closed-loop system or by inputs $u^{\prime}$ to the system with loop broken at point $\times \times$.

The first two properties are also the source of much of the temptation surrounding observers, however. We see that input/output properties are the same and even certain loop transfer functions are the same. The latter promise equal relative stability properties, equal tolerance to uncertainties (robustness), and equal disturbance rejection properties. What more could we ask for?

The problem, of course, is that the loop transfer properties are the same at point $\times \times$, inside our own control implementation where only masochists would insert significant uncertain elements or disturbances. According to Property 3, equal loop transfer characteristics are not obtained at the control signal interface to the plant, point $\times$, where nature gets to insert uncertainties and disturbances. It is at this point that robustness properties must be measured, and, as seen in [2], it is 


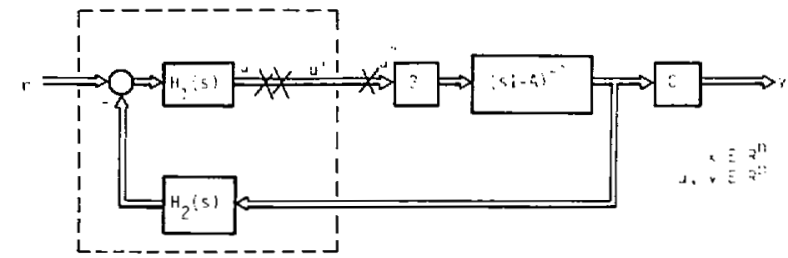

(a)

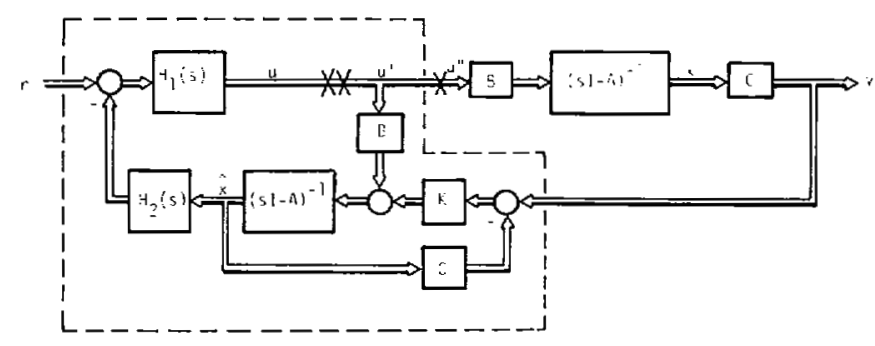

(b)

Fig. 1. (a) Full-state feedback implementation. (b) Observer-based inplementation.

here that observer-based implementations can fall well short of our objectives.

The fact that loop transfer functions will in general be different at point $x$ follows by noting that, unlike before, the observer error dynamics do get excited in response to inputs $u^{\prime \prime}$ with loops open at $X$. The more interesting fact is that such differences are avoided if (1) holds. This latter result is apparently not as well known, so a simple derivation is given in Appendix A. It is important because it offers a way to adjust observers so that full-state loop transfer characteristics are recovered at point $X$. In particular, suppose the observer gains are parameterized as a function of a scalar variable $q$. Let this function, $K(q)$, be selected such that as $q \rightarrow \infty$

$$
\frac{K(q)}{q} \rightarrow B W
$$

for any nonsingular matrix $W$. Then (1) will be satisfied asymptotically as $q \rightarrow \infty$. The resulting observer error dynamics will have limiting poles given by roots of the polynomial

$$
\psi(s)=\operatorname{det}(s I-A) \operatorname{det}\left[I+q C(s I-A)^{-1} B W\right] .
$$

$P$ of these roots will tend toward the $P$ finite transmission zeros of the plant, i.e., the zeros of polynomial

$$
\psi(s)=\operatorname{det}(s I-A) \operatorname{det}\left[C(s I-A)^{-1} B\right]
$$

which are stable by assumption, and the rest will tend to infinity. It is clear from this that the commonly suggested approach of making all roots of the error dynamics arbitrarily faster is generally the wrong thing to do.

\section{AN OBSERVER-AdJustment Procedure}

Equation (2) defines the required limiting characteristics of an adjustment trajectory $K(q)$ which changes arbitrary initial nominal observer gains, $K(0)$, with poor robustness properties into better gains asymptotically. We still need to define details of such trajectories.

A basic requirement for every point of an adjustment trajectory is stability of the observer error dynamics. Clearly, if we violate this requirement, overall closed-loop stability is also lost. (Note that this does not mean that the net compensator within the dashed lines of Fig. I(b) needs to be stable.) One way to assure stable error dynamics is to restrict the observer to be a Kalman filter for some set of noise parameters. That is, let

$$
K(q)=\Sigma(q) C^{T} R^{-1}
$$

with $\Sigma(q)$ defined by the Riccati equation

$$
A \Sigma+\Sigma A^{T}+Q(q)-\Sigma C^{T} R^{-1} C \Sigma=0 .
$$

As usual we take $Q=Q^{T}>0$ and $R=R^{T}>0$ with $\left(A, Q^{1 / 2}\right)$ and $(C, A)$ stabilizable and observable, respectively. For Kalman filters, these matrices represent given process noise and measurement noise intensities. Here they are treated more freely as design parameters which we can select to suit broader purposes. In particular, let

$$
\begin{aligned}
Q(q) & =Q_{0}+q^{2} B V B^{T} \\
R & =R_{0}
\end{aligned}
$$

where $Q_{0}$ and $R_{0}$ are noise intensities appropriate for the nominal plant and $V$ is any positive definite symmetric matrix. With these selections, the observer gain for $q=0$ corresponds to the nominal Kalman filter gain. However, as $q$ approaches infinity, the gains are seen from (5) to satisfy

$$
\frac{K R K^{T}}{q^{2}} \rightarrow B V B^{T}
$$

To show this formally, (5) with weights (6) can be divided by $q^{2}$ yielding

$$
A\left(\frac{\Sigma}{q^{2}}\right)+\left(\frac{\Sigma}{q^{2}}\right) A^{T}+\frac{Q_{0}}{q^{2}}+B V B^{T}-q^{2}\left(\frac{\Sigma}{q^{2}}\right) C^{T} R^{-1} C\left(\frac{\Sigma}{q^{2}}\right)=0 .
$$

It then follows from [5, p. 307] that

$$
\left(\frac{\Sigma}{q^{2}}\right) \rightarrow 0 \quad \text { as } q \rightarrow \infty
$$

whenever the transfer function $C(s I-A)^{-1} B$ has no right half-plane zeros. Consequently,

$$
q^{2}\left(\frac{\Sigma}{q^{2}}\right) C^{T} R^{-1} C\left(\frac{\Sigma}{q^{2}}\right) \rightarrow B V B^{T}
$$

and (8) is established. Solutions of (8) must necessarily be of the form

$$
\frac{1}{q} K \rightarrow B V^{1 / 2}\left(R^{1 / 2}\right)^{-1}
$$

where $V^{1 / 2}$ denotes some square root of $V$, i.e., $\left(V^{1 / 2}\right)^{T} V^{1 / 2}=V$ and similarly, $R^{1 / 2}$ is some square root of $R$. Since (12) is a special case of (2), it follows that the adjustment procedure defined by (4)-(7) will achieve the desired robustness-improvement objective.

Note that the second term in (6) can be interpreted as extra process noise added directly to the control input of the plant. Within the constraints of Kalman filter mathematics, such "fictitious noise" is a natural mechanism to represent uncertainties at this point of the control loop. It is nice to know that the resulting filter design actually responds with a corresponding robustness improvement. Note, however, that arbitrary increases of the existing noise matrix (i.e., $Q=\left(1+q^{2}\right) Q_{0}$ or addition of arbitrary full rank noise process (i.e., $Q=Q_{0}+q^{2} W$ with $W=W^{T}>0$ ) which are often suggested as other intuitive robustness improvement methods, will not in general produce the desired effect. This point is illustrated with an example in Section IV.

Finally, we note that the use of Kalman filter equations in the adjustment procedure is not fundamental. The filters merely provide a convenient way to define a $K(q)$ function which assures stability along the entire adjustment trajectory and has the desired limiting behavior (2). Any other procedure (pole placement, for example) with the same properties could be used as well. We emphasize, however, that both stability along the trajectory and asymptotic behavior must be achieved. Hence, such "obvious" choices as

$$
K(q)=q B W
$$

will only work for special systems which are stabilizable with high gain static output feedback alone. The Kalman filter choice (4), in contrast, works for all controllable, observable, minimum phase plants.

\section{AN Example}

To illustrate the observer properties and adjustment procedure above, consider the following example. 


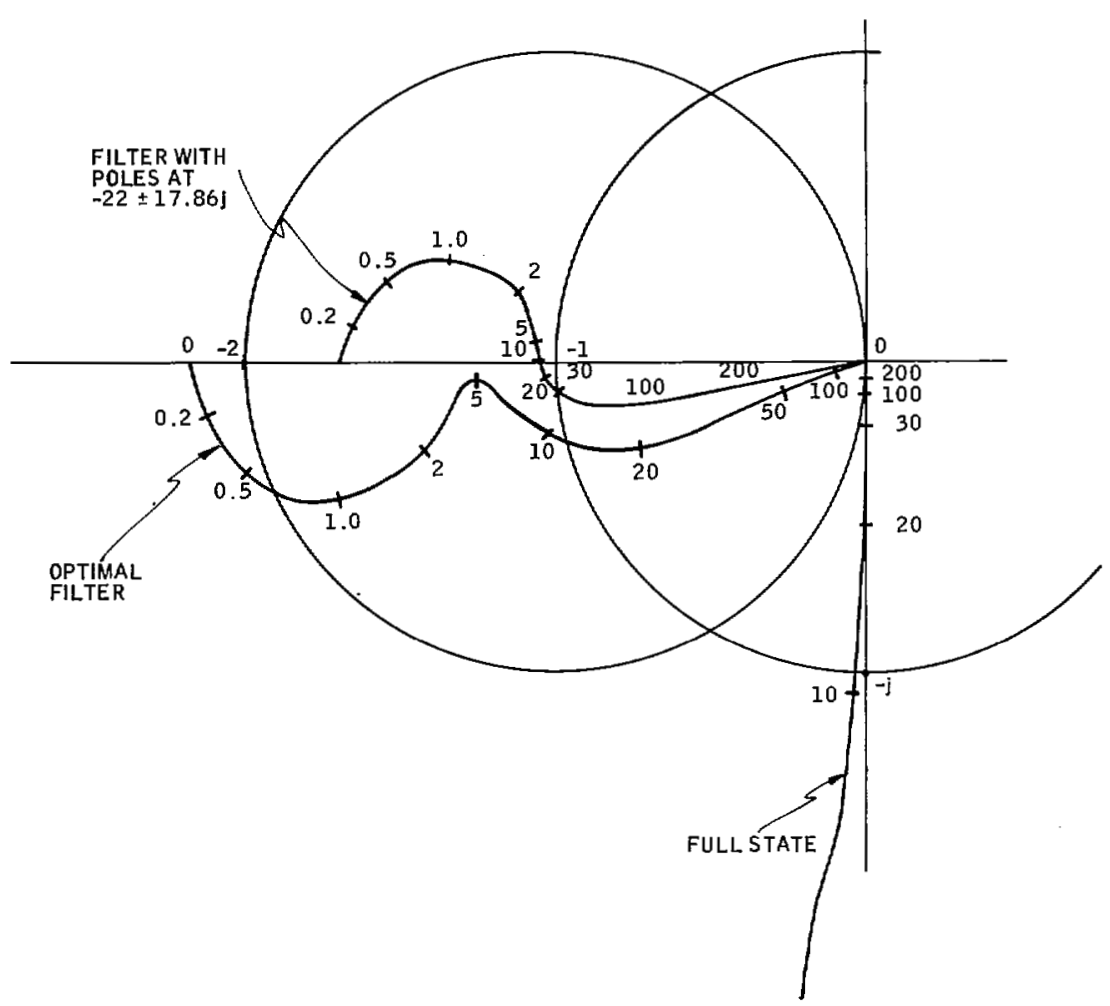

Fig. 2. Nyquist diagram of the optimal filter and observer.

Plant:

$$
\begin{aligned}
\frac{d x}{d t} & =\left[\begin{array}{rr}
0 & 1 \\
-3 & -4
\end{array}\right] x+\left[\begin{array}{l}
0 \\
1
\end{array}\right] u+\left[\begin{array}{r}
35 \\
-61
\end{array}\right] \xi \\
y & =\left[\begin{array}{ll}
2 & 1
\end{array}\right] x+\eta
\end{aligned}
$$

with $E(\xi)=E(\eta)=0 ; E[\xi(t) \xi(\tau)]=E[\eta(t) \eta(\tau)]=\delta(t-\tau)$. Controller:

$$
u=\left[\begin{array}{ll}
-50 & -10
\end{array}\right] \hat{x}+[50] r .
$$

The plant in this example is a (harmless) stable system with transfer function

$$
\frac{y}{u}(s)=\frac{s+2}{(s+1)(s+3)}
$$

The controller happens to be a linear-quadratic one, corresponding to the performance index

$$
J=\int_{0}^{\infty}\left(x^{T} H^{T} H x+u^{2}\right) d t
$$

with

$$
H=4 \sqrt{5}\left[\begin{array}{ll}
\sqrt{35} & 1
\end{array}\right] .
$$

It places the closed-loop regulator poles at

$$
s=-7.0 \pm j 2.0 \text {. }
$$

A Nyquist diagram (polar plot of the loop transfer function at point $x$ ) for the full-state design is given in Fig. 2. Gain margin is infinite in both directions and there is over $85^{\circ}$ phase margin. The design is then implemented using a Kalman filter for the given noise parameters. The Nyquist plot for the resulting observer-based controller is also shown in Fig. 2. Oops... less than $15^{\circ}$ phase margin.

In an effort to improve this margin, one adjustment to the filter that could be made is to speed it up. So, we can try moving the filter/observer poles to the left in a second-order Butterworth pattern. For the filter/observer poles at $-22 \pm 17.86 j$ one gets the third Nyquist plot in Fig. 2. As can be seen, the results are less than satisfactory. Not only are the margins disappearing (now less than $10^{\circ}$ ) but the loop bandwidth has increased (crossover has gone from approximately 12 to $40 \mathrm{rad} / \mathrm{s}$ ).
Unless we are trying to design an explosive device, this is clearly undesirable. It gets worse as the filter gets faster. In fact, it can be shown that the margins go asymptotically to zero for large gains, while the loop bandwidth goes to infinity. The present example is not a pathological one, either. Similarly, undesirable characteristics for fast filters are obtained with most systems.

When the observer adjustment procedure of Section III is applied to the same example, much more pleasing behavior is obtained. Following (6) $-(7)$, we let the process noise covariance matrix be

$$
Q=\left(\begin{array}{r}
35 \\
-61
\end{array}\right)\left(\begin{array}{ll}
35 & -61
\end{array}\right)+q^{2}\left(\begin{array}{l}
0 \\
1
\end{array}\right)\left(\begin{array}{ll}
0 & 1
\end{array}\right) .
$$

We then increase $q$ from zero until a reasonable compromise between noise performance and robustness is achieved. Some results of this process are summarized in Fig. 3 and Table I. Fig. 3 shows Nyquist diagrams for $q^{2}=100,500,1000$, and 10000 . Margins improved with essentially no change in bandwidth as the modified loop transfer function tends toward full-state optimal. Noise performance is summarized in Table I for the same set of $q$ values. As expected, the error covariance of the adjusted filter with respect to the original noise increases markedly with $q$. However, there was not the same deterioration in state covariance.

Table I also documents other parameters associated with these design points-poles of the error dynamics, margins, and filter gains. Note in particular that the filter poles tend toward the plant zero and toward infinity, as required by (3).

This adjustment procedure was also successfully applied to reconstruction of measured outputs after sensor failures for the A7-D aircraft [8]. In this application the optimum Kalman filter produced an unstable system when tested in hybrid simulation over the aircraft's flight envelope. After attempts with "ad hoc" fictitious noise adjustment procedures failed the method discussed here successfully stabilized the system. Also, the resulting error covariance properties remained close to the optimum values.

\section{ConClusions}

This paper illustrates some of the difficulties one can get into by relying on observers for state reconstruction. We have concentrated on robustness properties. In general, these will be poorer for observer-based 


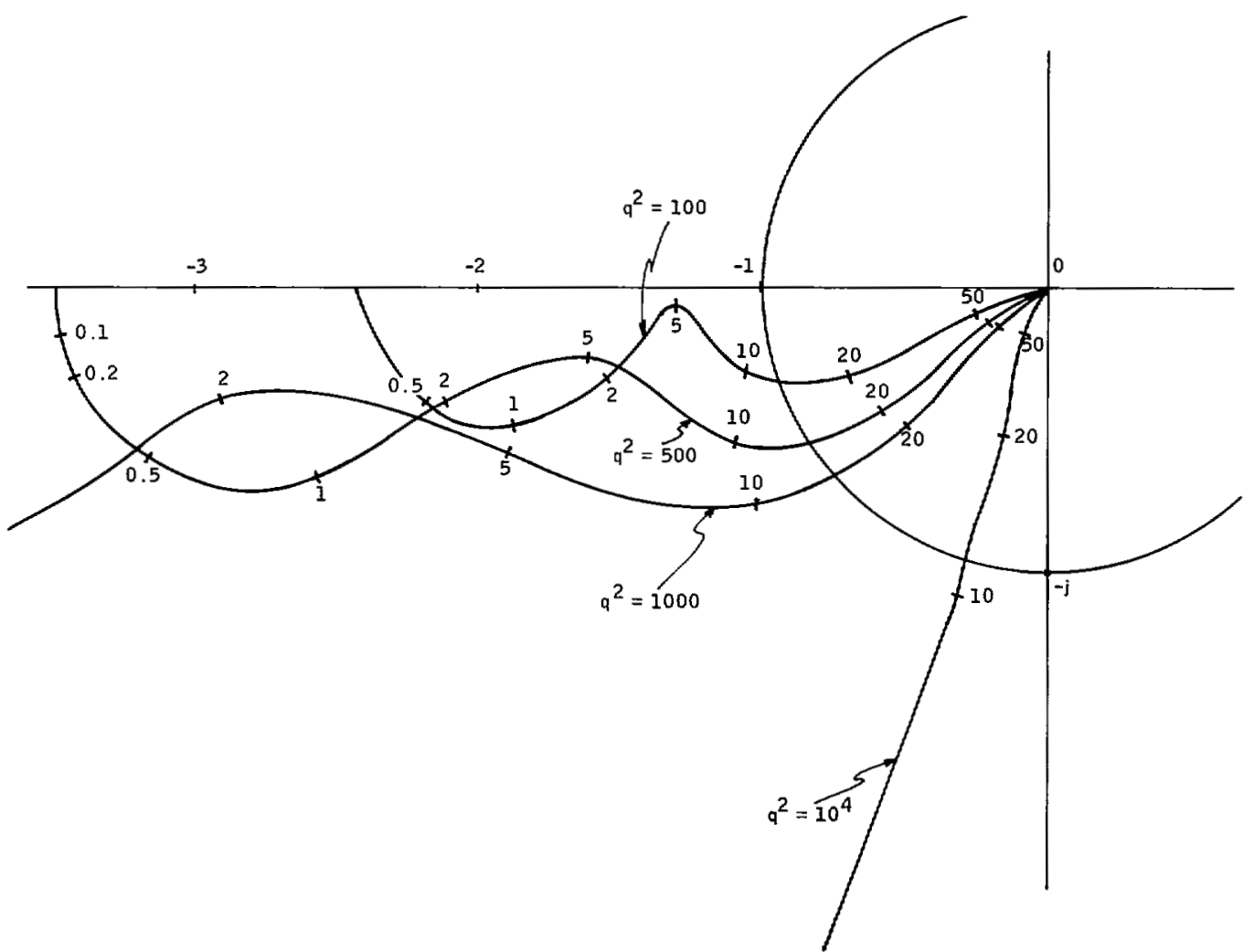

Fig. 3. Nyquist diagrams of the ficticious noise design procedure.

TABLE I

SUMMARY OF EXAMPLE

\begin{tabular}{|c|c|c|c|c|c|c|c|c|}
\hline & $\begin{array}{l}\text { FILTER } \\
\text { POLES }\end{array}$ & $\begin{array}{c}\text { GAIN } \\
\text { MARGIN } \\
\mathrm{db}\end{array}$ & $\begin{array}{c}\text { PHASE } \\
\text { MARGIN } \\
\text { deg }\end{array}$ & $\begin{array}{r}E R \\
\text { COVA } \\
E[(x-\end{array}$ & $\begin{array}{l}\text { OR } \\
\left.\text { IANCE }(x-\hat{x})^{\top}\right]\end{array}$ & & & $\begin{array}{c}\text { FILTER } \\
\text { GAIN }\end{array}$ \\
\hline $\begin{array}{l}\text { Optimal } \\
\text { LQG Design }\end{array}$ & $-7 \pm 2 j$ & -6.75 & 15 & $\begin{array}{c}97 \\
-163\end{array}$ & $\begin{array}{r}-163 \\
277\end{array}$ & $\begin{array}{r}221 \\
-613\end{array}$ & $\begin{array}{l}-613 \\
2070\end{array}$ & $\begin{array}{r}30 \\
-50\end{array}$ \\
\hline $\begin{array}{l}\text { Fast Fil ter Adjust- } \\
\text { ment Procedure }\end{array}$ & $-22 \pm 17.9 j$ & -.98 & 10 & $\begin{array}{r}6280 \\
-12200\end{array}$ & $\begin{array}{r}-12200 \\
23800\end{array}$ & $\begin{array}{r}130 \\
-613\end{array}$ & $\begin{array}{l}-613 \\
8520\end{array}$ & $\begin{array}{r}720 \\
-1400\end{array}$ \\
\hline $\begin{array}{l}\text { Fictitious Noise } \\
\text { Adjustment Procedure } \\
q^{2}=100\end{array}$ & $\begin{array}{r}-4.3 \\
-13.1\end{array}$ & -7.73 & 19 & $\begin{array}{r}107 \\
-184\end{array}$ & $\begin{array}{r}-184 \\
319\end{array}$ & $\begin{array}{r}236 \\
-613\end{array}$ & $\begin{array}{l}-613 \\
1810\end{array}$ & $\begin{array}{r}26.8 \\
-40.2\end{array}$ \\
\hline$q^{2}=500$ & $\begin{array}{l}-2,9 \\
-24\end{array}$ & -10.9 & 33 & $\begin{array}{r}163 \\
-301\end{array}$ & $\begin{array}{r}-301 \\
564\end{array}$ & $\begin{array}{r}268 \\
-613\end{array}$ & $\begin{array}{l}-613 \\
1500\end{array}$ & $\begin{array}{r}20.4 \\
-17.7\end{array}$ \\
\hline$q^{2}=10^{3}$ & $\begin{array}{l}-2.5 \\
-33\end{array}$ & -13.9 & 42 & $\begin{array}{r}204 \\
-385\end{array}$ & $\begin{array}{r}-385 \\
743\end{array}$ & $\begin{array}{r}285 \\
-613\end{array}$ & $\begin{array}{l}-613 \\
1360\end{array}$ & $\begin{array}{l}16.7 \\
-1.9\end{array}$ \\
\hline$q^{2}=10^{4}$ & $\begin{array}{l}-2.1 \\
-100\end{array}$ & -37 & 74 & $\begin{array}{r}290 \\
-570\end{array}$ & $\begin{array}{l}-570 \\
1170\end{array}$ & $\begin{array}{r}317 \\
-613\end{array}$ & $\begin{array}{l}-613 \\
1200\end{array}$ & $\begin{array}{r}6.9 \\
84.6\end{array}$ \\
\hline
\end{tabular}

implementations than for full-state implementations. For minimum phase systems, however, full-state robustness can be recovered asymptotically provided it is done correctly. Fast observers are not in general correct. A "fictitious noise" adjustment procedure was suggested which is correct.

The apparent practical value of this procedure is that it gives a simple way of trading off between noise rejection and margin recovery. When $q=0$, the filter will be optimal with respect to the "true" (as modeled) system noise. As $q$ increases the filter will do a poorer job of noise rejection but the closed-loop stability margins will improve. Hopefully, a satisfactory compromise can be found through the adjustment of the single parameter $q$. We stress that margin recovery occurs at point $\times$ in Fig. 1, at the control signal interface to the outside world. Asymptotically, the full-state and observer-based implementations will have the 
same tolerance to disturbances and uncertain elements inserted at this point. While point $x$ is clearly a physically important one (more important than point $\times \times$, certainly), engineers who may wish to test robustness at still other points in the control loop should recognize that the recovery results may not be applicable there. If such other points are judged more important than $\times$, a slight generalization of the adjustment procedure may be used to ensure margin recovery, as outlined in Appendix A.

The suggested adjustment procedure is essentially the dual of a sensitivity recovery method suggested by Kwakenaak [7]. The latter provides a method for selecting the weights in the quadratic performance index so that full-state sensitivity properties are achieved asymptotically as the control weight goes to zero. In this case, however, closed-loop plant poles instead of observer poles are driven to the system zeros, which can result in unacceptable closed-loop transfer function matrices for the final system.

APPENDIX A

DertVation OF Property 3

Referring to Fig. 1(a), the transfer functions from control signal $u^{\prime \prime}$ to states $x$ (with loop broken at point $X$ ) are given by

$$
x=\Phi B u^{\prime \prime}
$$

where $\Phi=(s I-A)^{-1}$. The corresponding transfer functions from $u^{\prime \prime}$ to $\hat{x}$ in Fig. 1(b) are

$$
\begin{aligned}
\hat{x}= & \left(\Phi^{-1}+K C\right)^{-1}\left(B u^{\prime}+K C \Phi B u^{\prime \prime}\right) \\
= & {\left[\Phi-\Phi K(I+C \Phi K)^{-1} C \Phi\right]\left(B u^{\prime}+K C \Phi B u^{\prime \prime}\right) } \\
= & \Phi\left[B(C \Phi B)^{-1}-K(I+C \Phi K)^{-1}\right] C \Phi B u^{\prime} \\
& +\Phi\left[K(I+C \Phi K)^{-1}\right] C \Phi B u^{\prime \prime} .
\end{aligned}
$$

We now note that (A.3) is identical to (A.1) if (1) is satisfied. Hence, all control signals based on $\hat{x}$ in Fig. 1(b) (e.g., $u^{\prime}=-H_{1} H_{2} \hat{x}$ ) will have identical loop transfer functions as the corresponding controls based on $x$ in Fig. 1(a) (i.e., $u^{\prime}=-H_{1} H_{2} x$ ). This completes the derivation.

We close with the final observation that the equivalence of (A.1) and (A.3) is a property which can be achieved for other loop breaking points in the plant instead of point $\times$. Consider an arbitrary point $Y$ with variables $\nu(\operatorname{dim}(\nu)=m)$, and let $\nu^{\prime \prime}$ denote inputs at point $Y$ with the loop broken at $Y$. Then a full state implementation has the transfer functions

$$
x=\Phi^{1}\left(B u+F \nu^{\prime \prime}\right)
$$

where $\Phi^{1}$ is the transfer matrix $\left(s I-A^{1}\right)^{-1}$, modified from $\Phi$ by the broken loops. $F$ is the control input matrix for point $Y$. The corresponding observer-based implementation has the transfer functions

$$
\hat{x}=\left[\left(\Phi^{1}\right)^{-1}+K C\right]^{-1}\left[B u+F \hat{\nu}+K C \Phi^{1}\left(B u+F \nu^{\prime \prime}\right)\right]
$$

Following steps analogous to (A.2)-(A.3), this reduces to

$$
\begin{aligned}
\hat{x}= & \Phi^{1} B u \\
& +\Phi^{1}\left[F\left(C \Phi F^{1} F\right)^{-1}-K\left(I+C \Phi^{1} K\right)^{-1}\right] C \Phi^{1} F \hat{\nu} \\
& +\Phi^{1}\left[K\left(I+C \Phi^{1} K\right)^{-1}\right] C \Phi^{1} F \nu^{\prime \prime} .
\end{aligned}
$$

We again note that (A.6) is identical to (A.4) if the following modified statement of (1) is satisfied:

$$
K\left(I+C \Phi^{1} K\right)^{-1}=F\left(C \Phi^{1} F\right)^{-1} .
$$

Hence, all loop transfer functions based in $x$ in the observer-based implementation will be identical to loop transfer functions based on $x$ in the full-state implementation. Like (2), (A.T) can be satisfied asymptoti- cally by a "fictitious noise" adjustment procedure whenever the broken loop system

$$
\begin{aligned}
& \dot{x}=A^{1} x+F^{\prime \prime} \\
& y=C x
\end{aligned}
$$

is controllable, observable, and minimum phase. Note, however, that asymptotic satisfaction of (A.7) will generally preclude satisfaction of (1). Hence, we can recover margins at point $X$ or point $Y$ but not at both points simultaneously.

\section{ACKNOWLEDGMENT}

We would like to thank the Math Lab Group, Laboratory for Computer Sciences, MIT, for use of their invaluable tool, MAcsYMA, a large symbolic manipulation language. The Math Lab Group is supported by NASA under Grant NSG 1323 and by DOE under Contract E(11-1)3070.

\section{REFERENCES}

[1] M. G. Safonov and M. Athans, "Gain and phase margin of multiloop LQG regulators," IEEE Trans, Automat. Contr., Apr. 1977.

[2] J. C. Doyle, "Guaranteed margins for LQG regulators," IEEE Trans. Automat. Contr., Aug. 1978.

[3] A. G. J. MacFarlane and N. Karcanias, "Poles and zeros of linear multivariable systems: A survey of algebraic, geometric, and complex variable theory," Int. $J$. Contr., pp. 33-74, July 1976.

[4] F. C. Schweppe, Uncertain Dynamic Systems. Englewood Cliffs, NJ: Prentice-Hall, 1973.

[5] H. Kwakernaak and R. Sivan, Linear Optimal Control Systems. New York: WileyInterscience, 1972.

[6] B. D. O. Anderson and J. B. Moore, Linear Optimal Control. Englewood Cliffs, NJ: Prentice-Hall, 197 I.

[7] H. Kwakernaak, "Optimal low-sensitivity linear feedback systems," Automatica, vol 5, p. 279, May 1969.

[B] T. B. Cunningham, J. C. Doyle, and D. A. Shaner, "State reconstruction for flight control reversion modes," presented at the 1977 IEEE Conf. on Decision and Control, New Orleans, LA, Dec. 1977.

\section{Some Relations Satisfied by Prime Polynomial Matrices and Their Role in Linear Multivariable System Theory}

\section{P. J. ANTSAKLIS, MEMBER, IEEE}

Abstract-A number of relations which are satisfied by prime polynomial matrices are derived and then used to study the polynomial matrix equation $B G_{1}+G_{2} A=V$ and to parametrically characterize the class of stabilizing output feedback compensators.

\section{INTRODUCTION}

The concept of right (left) primeness of two polynomial matrices, a generalization of the primeness of two polynomials, is one of the most important concepts of linear multivariable system theory because it is directly related to the concepts of controllability and observability [8], [11]. It is known that to any two minimal dual factorizations $B_{1}(s) A_{1}^{-1}(s)$ and $A^{-1}(s) B(s)$ of a transfer matrix $T(s)$, i.e., $T=B_{1} A_{1}^{-1}=A^{-1} B$, correspond four polynomial matrices $X_{1}(s), Y_{1}(s), X(s)$, and $Y(s)$, which satisfy $X_{1} A_{1}+Y_{1} B_{1}=I$ and $A X+B Y=I$ [8], [11]. When these (nonunique) matrices are being used in the literature, they are usually supposed to have been derived independently, by some process, and they do not satisfy any other relations than the above. If $X_{1}, Y_{1}, X$, and $Y$ are

Manuscript received November 27, 1978; revised February 28, 1979. Paper recommended by M. K. Sain, Past Chairman of the Linear Systems Committee. This work was supported in part by the National Science Foundation under Grant ENG 77-04119.

The author was with the Department of Electrical Engineering, Rice University, Houston, TX 77001. He is now with the Department of Computing and Control, Imperial College, London, England. 